\title{
Las fuentes del periodismo de investigación sobre el servicio de inteligencia CNI
}

\author{
Fernando RUEDA RIEU \\ Centro Universitario Villanueva (UCM) \\ frueda@villanueva.edu
}

Recibido: 30 junio de 2013

Aceptado: 19 de noviembre de 2013

\begin{abstract}
Resumen
Las limitaciones legales impuestas por la Ley de Secretos Oficiales de 1978 y por la Ley Reguladora del Centro Nacional de Inteligencia (CNI) de 2002 a los periodistas para informar sobre las actividades del servicio de inteligencia (CESID-CNI) no han impedido, desde la llegada de la democracia, que los medios de comunicación informen de sus actividades. Ha sido posible gracias a las diversas fuentes con que han contado los profesionales, mucho más numerosas de las que podrían pensarse en un simple acercamiento al tema. Un estudio sobre 265 noticias relevantes publicadas en los últimos 30 años permite sistematizar quiénes y qué cargos ocupan los informantes que, sabiendo que su colaboración es perseguible legalmente, han ayudado a los periodistas a sacar a la luz esas noticias.

Palabras clave: Fuentes; Periodismo de investigación; periodismo sobre servicios de inteligencia; CNI; espionaje.

\section{The sources of investigative journalism on Spanish service of intelligence CNI (National Intelligence Center)}

\begin{abstract}
Legal limitations imposed by the Official Secrets Act 1978 and the Law Regulating the National Intelligence Center (CNI) from 2002 to journalists to report on the activities of the intelligence service (CESID$\mathrm{CNI}$ ) have not stopped since the arrival of democracy, the media report on their activities. It has been possible thanks to the various sources available to these professionals, much more numerous than they could think of a simple approach to the subject. A study of 265 relevant news published in the last 30 years permits to systematize all the information on the informants (who they are, their job status, etc.), knowing that their collaboration is legally prosecutable, has helped journalists to break the news.
\end{abstract}

Keywords: Sources; Investigative Journalism; journalism intelligence services; Spanish CNI; espionage.

\section{Referencia normalizada}

RUEDA RIEU, Fernando (2014): "Las fuentes del periodismo de investigación sobre el servicio de inteligencia CNI”. Estudios sobre el Mensaje Periodístico. Vol. 20, Núm. 1 (enero-junio), págs.: 539-555. Madrid, Servicio de Publicaciones de la Universidad Complutense.

Sumario: 1. Introducción. 2. Periodismo de investigación; 2.1. Las fuentes oficiosas; 2.2. La protección de las fuentes; 2.3. Confirmación de datos. 3. El Periodismo de investigación y las fuentes en los temas del servicio de inteligencia; 3.1. Los agentes y ex agentes; 3.2. El Gobierno; 3.3. Los parlamentarios; 3.4. Gabinete de prensa del CNI; 3.5. La cúpula del CNI; 3.6. Colaboradores e informantes; 3.7. Fuentes aledañas; 3.8. Fuentes materiales. 4. Conclusiones. 5. Referencias bibliográficas.

\section{Introducción}

Desde el inicio de la democracia en España han existido limitaciones legales importantes del servicio de inteligencia al trabajo de los periodistas, recogidas en la Ley de Secretos Oficiales y en la Ley Reguladora del CNI. Sin embargo, estas trabas no han impedido en ningún momento el control por parte de los periodistas de las actividades del servicio de inteligencia. 
El periodismo de investigación sobre los servicios de inteligencia es una materia de la que únicamente existe una tesis doctoral y nada de material científico, debido posiblemente al oscurantismo del CNI, anteriormente llamado CESID (Centro Superior de Información de la Defensa), y al reducido número de profesionales que tratan habitualmente este tipo de temas. A pesar de la relevancia del tema, hay que tener en cuenta la dificultad de su estudio dada la necesidad de los informadores de ocultar la identidad de sus fuentes. Sin embargo es un tema relevante académicamente por la necesidad de conocer cómo se construye el conocimiento en esta parcela. Además, para los profesionales es un tema necesario para saber cómo funcionan en la realidad estas investigaciones. Finalmente, es un asunto de interés divulgativo porque la opinión pública tiene el derecho a conocer cómo se montan informaciones que frecuentemente son motivo de escándalo en la sociedad.

El estado de la cuestión justifica ampliamente la necesidad de esta investigación, pues hasta el momento no se ha producido ninguna aportación científica sobre el tema de las fuentes en los temas de espionaje, más allá de una tesis doctoral en Periodismo que toca el tema de las fuentes sin entrar en profundidad. Existe una legislación claramente limitativa del trabajo de los periodistas, pero el estudio de los casos seleccionados permitirá sacar a la luz importantes conclusiones. Casos que son una base teórica suficiente para generar una investigación.

El objeto del presente trabajo ha sido estudiar cuáles son las fuentes que facilitan a los profesionales de la información esas noticias. En muchos casos resulta factible descubrir la identidad de las mismas y en la mayoría identificar la institución para las que trabajan.

Partíamos de la hipótesis de que la mayor parte de los periodistas habían obtenido sus informaciones relacionándose con fuentes oficiosas que serían agentes y ex agentes, aunque aceptábamos la posibilidad de que desde otras instituciones o grupos de personas vinculadas con los asuntos de inteligencia también podían colaborar. La experiencia del autor como periodista especializado en la materia durante muchos años fue de gran utilidad a la hora de señalar esas posibles fuentes.

Hemos utilizado como método científico el análisis cualitativo y de contenido de las informaciones publicadas. Partiendo del análisis de contenido como "un método que busca descubrir la significación de un mensaje, ya sea este un discurso, una historia de vida, un artículo de revista..." (Gómez, 2000: web), nos hemos introducido en el análisis cualitativo siguiendo las apreciaciones de Ruiz Olabuénaga (2012: 24): "Opera en dos dimensiones y de forma circular. No solo se observan y graban los datos, sino que se entabla un diálogo permanente entre el observador y lo observado, entre inducción (datos) y deducción (hipótesis), al que acompaña una reflexión analítica permanente entre lo que se capta del exterior y lo que se busca cuando se vuelve, después de cierta reflexión, de nuevo al campo de trabajo".

Para conseguir el objetivo, analizamos 265 acontecimientos que fueron noticia en la prensa, reflejados en 7.950 elementos, publicados entre los años 1983 y 2013, referidas a las actividades del CNI y el CESID. Además, analizamos las informaciones de 3 diarios digitales y 28 libros que trataban sobre las actividades del servicio de inteligencia. Posteriormente, procedimos a analizar los elementos que cualitativamente 
más se ajustaban a la presente investigación y redujimos la lista a 20 noticias, que aparecían recogidas en los diarios El Mundo, El País y ABC; el semanario Tiempo; los diario digitales www.elpais.com, www.elmundo.es y www.elconfidencialdigital.es; y cinco libros.

A partir de este análisis se han identificado casos paradigmáticos de la relación fuentes-periodista: la dimisión del director del CNI Alberto Saiz; "Nota Interior" del CESID sobre la muerte de la etarra Urigoitia; la reunión de Carod Rovira con dirigentes de ETA; la desclasificación de documentos del CNI tras el atentado del 11-M; comparecencia en el Congreso del director Félix Sanz para hablar de Método 3; la visita de Xabier Arzalluz a la sede del CNI; la entrega de una condecoración a Mikel Lejarza; el cese de Cassinello como Director Técnico de Inteligencia; el plan del director Calderón para cambiar la imagen del CESID; el chantaje al ciudadano marroquí Ouazzani; la detención de una funcionaria de la Seguridad Social al servicio del CESID; los casos de espionaje en la década de los 80 a altas autoridades del Estado; el apoyo de un espía a los escuadrones de la muerte en El Salvador; el "Manual de Inteligencia" publicado íntegramente en un semanario; el espionaje a Adolfo Suárez cuando era presidente del Gobierno; investigación sobre la vida privada y pública del ex agente Perote; y el alquiler de la vivienda del ministro Serra por una empresa tapadera del CESID.

\section{Periodismo de investigación}

¿Cómo conseguir penetrar en las alcantarillas del poder de la que forman parte los servicios de inteligencia, si los responsables del mismo, tanto políticos como profesionales, hacen todo lo que está en sus manos para evitar que se conozcan sus desviaciones de poder y han tendido un manto de silencio, incluso presionando tácita o abiertamente a los que pudieran conocer algún aspecto del comportamiento irregular?

La respuesta está en el periodismo de investigación. Caminos Marcet (1997: 17) explica que "el periodismo de investigación tiene un sello de identidad propio, caracterizado porque va más allá y busca esa información que no circula por los cauces normales. Es un periodismo profundo, que exige tiempo y dedicación, búsqueda constante". Pepe Rodríguez (1994: 23) define al periodista investigador como aquel que "utilizando técnicas habituales de la profesión u otras específicas y/o habitualmente atribuibles a profesiones ajenas a la suya, elabora una información producto de un número indeterminado de fuentes (atribuibles o no) y de un análisis personal de datos, contrastados con mayor o menos eficacia, que le conducen a comunicar una noticia sobre una realidad que, por su propia configuración y naturaleza, estaba destinada a permanecer oculta durante un periodo de tiempo indefinido".

Petra Secanella (1986: 34) recoge los tres requisitos trascendentales para considerar un trabajo encuadrado en el periodismo de investigación: Que sea el resultado del trabajo del periodista, no la información elaborada por profesionales como la Policía; que el objeto de la investigación sea razonablemente importante para un gran sector de la población; y que los investigados intenten esconder esos datos al público.

Carlos Soria (1997: 180) prefiere hablar de periodismo de denuncia, pues defiende que "todo el periodismo digno de este nombres siempre es periodismo de investiga- 
ción”. En esta línea aporta una nueva visión sobre su campo de actuación al especificar que "versa sobre hechos o actuaciones ilegales o no éticas producidas en el ámbito público, que el poder político, el poder económico o los poderes sociales no tienen interés alguno en que sean públicamente conocidos".

La trascendencia del periodismo de investigación queda reflejada en el estudio realizado por el profesor norteamericano Silvio Waisbord (2001: web), quien explica que "el reportaje producto de investigación es una de las contribuciones más importantes de la prensa a la democracia", señala que "el periodismo puede contribuir al rendimiento de cuentas mediante la vigilancia del funcionamiento de las instituciones" y defiende que "la prensa ha mantenido vivas las alegaciones de conducta ilegal o poco ética y, a la postre, en algunos casos, ha forzado a los organismos legislativo y judicial a actuar".

Conviene hacer una importante matización. A veces lo que se publica en España sobre los servicios de inteligencia no es periodismo de investigación, sino periodismo de filtración que busca mejorar la imagen del servicio de inteligencia. Ramón Reig (2000: 102) cuenta el caso de un reportaje emitido por Antena 3 en abril de 1994, bajo la etiqueta de un programa especial del equipo de investigación de la cadena. Señala que el reportaje estaba elaborado muy certeramente, con una presentación formal muy cuidada, llena de misterios, en el que se observaban puertas que se cierran y caras en la sombra de agentes del CESID. La conclusión de este experto es clara: el programa tuvo "una buena puesta en escena pero nada de investigación. Muy al contrario, a veces da la impresión de que se trata de un reportaje promocional".

\subsection{Las fuentes oficiosas}

El tema de las fuentes es trascendental en el periodismo. El de información se caracteriza por utilizar generalmente fuentes abiertas y habitualmente disponibles para los periodistas. El periodismo de investigación utiliza esas mismas fuentes y otras no atribuibles. Estas últimas pertenecen a ámbitos habitualmente no accesibles al periodista corriente. Son las fuentes oficiosas, que Pepe Rodríguez (1994: 97) define como "preferentemente de orden general, confidencial, tanto voluntaria como involuntaria e igualmente asidua como ocasional". También habla de que la información que facilitan estas fuentes es singularizada y original y que no suele asumir la paternidad de la misma.

Esas fuentes pueden desvelar al periodista informaciones que violen la Ley de Secretos Oficiales y la Reguladora del CNI. En ese caso, como en todos los demás, el periodista deberá aplicar escrupulosamente los principios generales del periodismo de investigación, y valorar si la información es de interés para la opinión pública, si habla de un comportamiento ilegal que alguien trata de ocultar y conseguir que en todo momento la iniciativa de la investigación le pertenezca a él. Este proceso implica que los daños que se produzcan -normales tras la difusión de una investigación- afecten a personas e instituciones, pero no perjudiquen auténticamente a los intereses del Estado.

En los últimos años, de una forma creciente, el análisis de contenido de las informaciones muestra cómo hay una excesiva tendencia en los periodistas a no especificar la identidad de sus informantes. El Observatorio chileno de Medios Fucatel (Munizaga, 2008: web) bautiza irónicamente a este fenómeno como el "Señor Fuen- 
tes", en referencia a este tipo de enunciados que "alude ambigua y genéricamente al origen de una información, ocultando la verdadera identidad del hablante: su nombre, su cargo u oficio, su calificación y experiencia y, más relevante aún, su confiabilidad e idoneidad".

Frente a esta crítica referida al periodismo de información, la Defensora del Lector del diario El País (Pérez Oliva, 2010: web) reconoce que se pueden encontrar en las páginas de su diario, con más frecuencia de lo deseable, crónicas y reportajes "basados en fuentes anónimas cuya ocultación no está en absoluto justificada". Y añade que este tipo de fuentes son imprescindibles en periodismo y explica que "es imposible hacer buen periodismo de investigación sin tener que recurrir en ocasiones a fuentes que exigen permanecer en el anonimato".

El tema de las fuentes oficiosas siempre ha sido motivo de debate. José Luis Dader (2002: 26) explica que "ni siquiera en el caso extremo de una "garganta profunda" que por su propio interés busque al informador para suministrarle unos datos o revelaciones, cabe confundir el auténtico periodismo de investigación con la simple aceptación de la intoxicación".

\subsection{La protección de las fuentes}

Las fuentes de los temas de servicios de inteligencia son especialmente sensibles al necesario secreto profesional y al "off the record". El informador se encuentra con frecuencia en la necesidad de estar pendiente de protegerlas. El periodista de investigación debe hacer saber a su fuente que si la situación llega al extremo de que un juez cita al periodista y le pide que revele su identidad, se negará a identificarlo, llegando incluso a preferir la cárcel antes de faltar a su deber profesional y al acuerdo alcanzado. Acción que repercutirá en el propio beneficio del investigador, que al garantizar el anonimato conseguirá que el informante se sienta más libre para desvelar todo lo que sabe. Especialistas en Derecho a la Información como María Pilar Cousido (1994: 42) defienden la necesidad de respetar el secreto profesional y hacerlo compatible con la Ley de Secretos Oficiales.

En algún caso se ha llegado a probar que por filtrar información una fuente ha sido amenazada de muerte. Es el caso denunciado en la revista Tiempo (Rueda, 1995: 16) bajo el título de "Un asesino a sueldo, listo para matar a Perote". La historia desvelaba que el ex agente del CESID había recibido amenazas para acabar con su vida tras filtrar documentos que probaban unas escuchas ilegales del servicio secreto al Rey, y a otras personalidades de la vida pública.

El caso más representativo en la historia del periodismo sobre protección a una fuente tuvo lugar en 1972 a raíz del "caso Watergate". Bob Woodward y Carl Bernstein, comenzaron investigando la entrada clandestina de unos ladrones en la sede del Partido Demócrata. Después, poco a poco, llegaron a la conclusión de que ese robo formaba parte de una campaña mucho más amplia del Partido Republicano para desprestigiar a sus oponentes.

Nadie sabía de dónde procedía la información tan importante que iban publicando. Posteriormente se descubrió que tenían un informador bien colocado en la cúpula del gobierno. Le bautizaron como "garganta profunda", pero siempre se negaron a des- 
velar su identidad para evitarle cualquier tipo de represalia. Unos cuantos años después, el profesor de Periodismo y premio Pulitzer, Clark Mollenhoff (Fernández, 1992: 3) consideró que "la utilización de "Garganta profunda" no fue muy correcta porque probablemente no era una prueba directa, sino de segunda o tercera mano. Sin embargo, Woodward y Bernstein compensaron ese defecto practicando una investigación sistemática".

Mollenhoff estaba equivocado, pero ninguno de los periodistas que conocía la identidad de la fuente le corrigió. Tuvieron que esperar casi 33 años para que finalmente se divulgara oficialmente quién era. El hombre tenía ya 91 años y una frágil salud. Se llamaba Mark Felt y era el número dos del FBI cuando tuvo lugar el escándalo. Desde ese puesto, tenía acceso a toda la información reservada sobre lo que estaba pasando en Estados Unidos.

Felt tenía argumentos para el resentimiento (Del Pino, 2005: 8): esperaba que le nombraran director del FBI, pero el presidente Nixon optó por un político amigo. Él, reconociendo lo anterior, especificaba que su obligación era impedir la presencia de un político corrupto en la Casa Blanca.

\subsection{Confirmación de datos}

Aunque las fuentes que utilice el periodista sean de la máxima credibilidad y confianza, la investigación siempre impone la exigencia de contrastar los datos aportados, para evitar que de una forma consciente o inconsciente toda la información o una parte de ella termine resultando falsa.

El periodismo anglosajón utiliza desde el éxito periodístico del citado "caso Watergate" la teoría de las tres fuentes: toda información para poder ser publicada tiene que estar apoyada en tres testimonios distintos, de personas fiables, sin intereses contra el objeto de la investigación y necesariamente desconectadas entre ellas.

Aunque un periodista investigador como Pepe Rodríguez (1994: 107) reconoce que "mientras no se obtenga esta triple confirmación, el periodista debería poner en duda el dato y no utilizarlo", él mismo y otros profesionales y estudiosos coinciden en que la teoría de las tres fuentes puede servir como referencia teórica, pero no como práctica habitual.

El periodista investigador trabaja en la verificación de una forma distinta en cada caso. A veces dos fuentes distintas le ofrecen la suficiente garantía para pasar a la publicación, porque considera suficientemente probada la información y contrastados los datos. En muchas ocasiones no llega a publicar la información, a pesar de contar con más de tres fuentes porque no está plenamente seguro de lo que tiene entre manos o porque no podría sustentarlo ante un juez. Aquí el periodista se plantea la prueba del algodón: si un juez le llamara y tuviera que probar la veracidad de la noticia, descontando las fuentes anónimas cuya identidad no puedo desvelar, ¿dispondría de suficientes pruebas?

Caminos Marcet (1997: 150) cita al periodista de investigación José María Irujo para añadir un nuevo dato. Afirma que muy raras veces se dispone de tres fuentes distintas y que suele ser suficiente disponer de dos. En algunos casos, cuando el periodista tiene la posibilidad de acceder a documentos vitales "o la fuente es seria, rigurosa, 
honrada [...] actúa por conciencia o porque el periodista ha tomado la iniciativa, podemos publicar el tema con una sola confirmación".

\section{El periodismo de investigación y las fuentes en los temas del servicio de inteli- gencia}

El periodismo de investigación sobre servicios de inteligencia se enfrenta a más limitaciones que cualquier otra especialidad. Las más importantes tienen que ver con la Ley 9/1968, de 5 de abril, de Secretos Oficiales, parcialmente modificada por la Ley 48/1978, de 7 de octubre. En su artículo 2 se establece que son materias clasificadas las que así sean expresamente declaradas por la ley y se trate de "asuntos, informaciones, documentos, datos y objetos cuyo conocimiento por personas no autorizadas pueda dañar o poner en peligro la seguridad y la defensa del Estado".

Un texto lo suficientemente amplio para abarcar casi cualquier información referida al CNI o a su antecesor el CESID. Es una restricción que atañe tanto al periodista como a sus fuentes y que en general limita la difusión de informaciones que pongan en riesgo lo llamado genéricamente "seguridad nacional", en aplicación de la "razón de Estado".

Más clara y directa, en este caso dirigido a las fuentes, es la Ley 11/2002, de 6 de mayo, reguladora del Centro Nacional de Inteligencia, que en su artículo 5.1 sobre actividades del CNI establece que son secreto todas sus actividades, su organización y estructura interna, medios y procedimientos, personal, instalaciones, bases y centros de datos, fuentes de información y las informaciones o datos que puedan conducir al conocimiento de las anteriores materias.

Estas prohibiciones imposibilitarían casi en su totalidad que ninguna fuente que se atuviera a la ley hablara con los periodistas para facilitar cualquier tipo de información sobre los excesos o comportamientos inmorales, ilegales o ilegítimos de personas que trabajan en el CNI, de la propia institución como tal o de sus mandos políticos.

\subsection{Los agentes $y$ ex agentes}

Una parte importante de las fuentes son los agentes, oficiales de inteligencia o espías -según las diversas terminologías- y los ex agentes, que comparten con sus compañeros en activo las mismas limitaciones legales para comunicarse con periodistas, igual que tuvieron en su día el mismo acceso a la información. Todos ellos tienen que rubricar antes de ser admitidos un compromiso de confidencialidad.

Las razones que llevan a un agente en activo a facilitar información a un periodista, violando la norma que con toda claridad se lo impide, pueden ser muy variadas. Intereses personales, motivos de conciencia, ansias de venganza...El hecho es que puede que la fuente tuviera algún problema, que sus motivaciones en la difusión de la historia sean espurias, pero el periodista debe valorar si la información habla de comportamientos ilícitos, inmorales o ilegales, proceder a confirmarla y ampliarla, para después publicarla siempre que sea de interés general. Sacar a la luz ese tipo de comportamientos es una obligación para los periodistas de investigación.

El 2 de julio de 2009, el entonces director del CNI, Alberto Saiz, presentó su dimisión ante las informaciones difundidas en los medios de comunicación referentes 
a sus comportamientos privados durante el ejercicio de su mandato. Tres meses antes, el diario El Mundo (Rubio, 2009a: 6) había empezado a publicar una investigación en la que denunciaba que Saiz había utilizado recursos y fondos públicos para su uso personal y disfrute durante sus viajes al extranjero.

En junio de ese año, poco tiempo después de que el servicio de inteligencia y su director no consiguieran evitar que siguieran apareciendo informaciones sobre la presunta corrupción (Rubio, 2009b: 4), diez agentes fueron "invitados" a someterse a una prueba del polígrafo. Tres rehusaron ateniéndose a la imposibilidad de obligar a los agentes a someterse al veredicto de la llamada "máquina de la verdad". La difusión de esta noticia (García Abadillo, 2009a: 1) demostró lo que ya se sospechaba: las filtraciones de las irregularidades de Saiz sólo podían proceder de agentes en activo con acceso a los documentos internos. El análisis poligráfico no fue concluyente. Pero que no pudieran acusar directamente a algunos de ellos no impedía que existiera la certeza de que la fuente o fuentes estaban dentro del servicio de inteligencia.

El propio Saiz denunció ante la Comisión de Secretos Oficiales del Congreso que la filtración a la prensa de las informaciones contra su persona eran una venganza de agentes del antiguo CESID (Sánchez, 2009: 1). Versión en línea por la aportada en varios medios, que hablaban de agentes descontentos, pues en sus cinco años de mandato al frente del CNI había cesado a más de 30 altos cargos. Fuera o no esta la razón, el hecho es que la información era de interés general y sirvió para sacar a la luz un comportamiento poco ético.

Un caso similar, pero protagonizado por un ex agente, se produjo años antes, en 1995. La reproducción de la "Nota Interior" del CESID con número "KA/5104/03-1187", produjo un enorme revuelo político (Cerdán y Rubio, 1996: 1). El informe señalaba que agentes de la Guardia Civil habían manipulado algunas pruebas del caso judicial para simular que la muerte de la etarra Lucía Urigoitia en 1987, durante el asalto al domicilio donde estaba escondida, se había producido en un tiroteo y no en un asesinato a sangre fría. La información sobre esa actuación ilegal procedía, según se supo posteriormente, de las microfichas que Juan Alberto Perote, ex jefe de la unidad operativa del CESID, se había llevado antes de abandonar su destino. Había sido el antiguo oficial de inteligencia el que había filtrado esa información voluntariamente a los periodistas.

\subsection{El Gobierno}

Los agentes y ex agentes no son las únicas fuentes posibles en el periodismo de investigación sobre servicios de inteligencia. El CNI envía sus informes al presidente del Gobierno y al vicepresidente, de quienes depende directamente, y a los ministros a los que puede interesar o convenir el conocimiento de una parte de ellos. Los más habituales en recibir esos documentos son Asuntos Exteriores, Defensa, Interior y, en los últimos tiempos tras la actual crisis, Economía.

El 26 de enero de 2004, el diario $A B C$ publicaba en su portada una noticia inquietante (Molina, 2004: 1): "Carod Rovira ultima un pacto con ETA para que no perpetre atentados en Cataluña", a la que acompañaban los datos de la reunión del dirigente de ERC y Conseller en Cap de la Generalitat con dos miembros de la cúpula de la or- 
ganización terrorista en Perpiñán. La información desató una crisis en el ejecutivo catalán. Carod Rovira denunció que había sido una maniobra del gobierno del presidente Aznar para acabar con él. La noticia, procedente de un informe del CNI, obligó al político a presentar la dimisión de su cargo en el ejecutivo catalán. Posteriormente, toda la oposición coincidió en acusar al gobierno de la Nación de utilizar los servicios secretos para atacar a ERC (Díez, 2004: web).

Un caso mucho más claro fue el que tuvo lugar el 18 de marzo de 2004, pocos días después del atentado del 11-M en Madrid. El Consejo de Ministros decidió desclasificar varios documentos del CNI para intentar demostrar que no había mentido durante la crisis posterior al estallido de las bombas en varios trenes. A pesar de que en los años anteriores tanto el gobierno del PSOE como el del PP se habían negado a enseñar papeles del servicio secreto alegando razones de seguridad, en este caso se saltaron la norma buscando un beneficio político propio (D.M. y J.P., 2004: 12).

\subsection{Los parlamentarios}

Otras fuentes del tema son los parlamentarios que forman parte de la Comisión de Secretos Oficiales del Congreso de los Diputados, cuya denominación actual es la de Comisión de Fondos Reservados. Su objetivo es que dentro de las cuatro paredes de la sala donde se reúnen, los miembros competentes del gobierno y el secretario de Estado Director del CNI expliquen temas sensibles a un reducido grupo de diputados. Baste señalar, para entender la importancia que se da a esta comisión, que los pequeños grupos nacionalistas más radicales no han conseguido hasta la fecha que sus representantes sean aceptados como miembros.

Todos los integrantes de la comisión acuden sabiendo que están obligados a guardar silencio sobre lo que allí escuchen, pero no ha habido ni una sola vez en la que la información allí transmitida no haya llegado a los medios inmediatamente. Así ocurrió el 19 de marzo de 2013. El director del CNI, Félix Sanz, compareció para hablar sobre su investigación sobre la princesa Corinna y su vinculación con la agencia de detectives Método 3. Al día siguiente, todos los medios de prensa desvelaban lo que dijo. El diario El País relataba que Sanz había acusado a la Generalitat de intentar identificar a sus agentes (González, 2013: 17) y el periodista Javier Fumero aseguraba en El Confidencial Digital que "los asistentes a este foro nunca guardan la debida reserva" (2013: web).

Los parlamentarios son también fuentes en otras ocasiones. Algunos diputados, que tienen entre sus competencias el control de los servicios secretos, reciben directamente del gobierno información de cierta calidad. Es el caso de Iñaki Anasagasti, del PNV, diputado en las primeras legislaturas y posteriormente senador. En un libro publicado en 2004 (Anasagasti, 2004: 255) cuenta sin tapujos su visita y la de Xabier Arzalluz, por entonces presidente del partido, a la sede del CNI y explica con todo lujo de detalles la identidad y los alias de los agentes con los que trató y la información que le contaron. Por ejemplo, saltándose lo prohibido por la ley, menciona a "José María Zaldua Mur, alias "Zaldivar", teniente coronel de Infantería de Marina". 


\subsection{Gabinete de prensa del CNI}

Tras la década de los 90, y en algunos países desde antes, servicios de inteligencia como el BND alemán crearon sus propios gabinetes de prensa y relaciones públicas para relacionarse con los medios de comunicación. Oficialmente pretendían hacer frente al oscurantismo que tanto se les criticaba en la sociedad. Y, de paso, también les sirvió para levantar un organismo que se relacionara directamente con los medios, facilitándoles informaciones abiertas -porcentaje de civiles y militares, actos en los que intervendrá el director...- y al mismo tiempo poder conocer con anterioridad cuáles eran los temas sobre los que iban a informar.

En España existe el Gabinete de Prensa del CNI, que inicialmente fue llevado por agentes más o menos cercanos a la información periodística y que desde la llegada de Félix Sanz a la dirección en 2009, tiene como director a un civil. Su trabajo y el de sus colaboradores consiste en recibir las peticiones de los medios de comunicación, notificarles que las van a poner en conocimiento de sus jefes y, cuando el tema es conflictivo, negar la contestación. También filtran información cuando corresponde a los intereses del servicio de inteligencia, como cualquier otro gabinete de prensa.

Son muchas las noticias en las que aparecen citados genéricamente los miembros del gabinete de prensa. Un ejemplo claro tuvo lugar a raíz de la concesión de una medalla a Mikel Lejarza, un agente que estuvo infiltrado en ETA. El articulista "El Chivato" de El Confidencial Digital (2010: web) afirma que ha preguntado sobre el tema y "la respuesta de un portavoz oficial ha sido que ni confirma ni desmiente la ceremonia. Una respuesta que hay que interpretar como una confirmación".

Algunos acreditados testimonios de primera mano muestran el verdadero fin de estos gabinetes de prensa. Sainz de la Peña (2001: 33), profesor de la Facultad de Ciencias Políticas y ex agente del CESID, afirma que la relación entre ambos estamentos adopta dos formas. "La primera es la de cobertura", en la que el servicio utiliza a la prensa para proporcionar cobertura a un agente -bien sea este un verdadero periodista reclutado por el servicio, bien un agente provisto de una acreditación de prensa- o como infraestructura operativa -caso de las delegaciones en el extranjero de las agencias de noticias nacionales-. "La segunda forma es la de la manipulación, cuando el servicio usa a la prensa para manipular la información", con una gama de actuaciones que van desde la censura hasta la difusión de las noticias falsas. El ex agente deja el concepto claro: los servicios de inteligencia ven a los periodistas como una herramienta para conseguir sus fines.

La misma tesis sobre la manipulación de los periodistas como fin primordial de los servicios de inteligencia la defiende el profesor Seijas (2001: 77), que ha conocido profesionalmente estos temas: "La CIA es consciente de que no hay más remedio que convivir con los medios de comunicación y si se puede, servirse de ellos". La pregunta es cómo hacerlo. La respuesta son los mencionados departamentos de "relaciones institucionales", bajo los que se esconden una serie de actividades, la principal de las cuales es "captar periodistas que trabajen para ellos y formar coberturas en todo el mundo sin levantar sospechas". 


\subsection{La cúpula del CNI}

Al margen del gabinete de prensa, las fuentes oficiales más productivas que buscan los periodistas están en la cúpula del servicio secreto. La periodista Pilar Cernuda ha reconocido públicamente sus comidas informativas con el director Emilio Alonso Manglano (Bardavío "et al", 2000: 247), otros han hecho lo mismo con el secretario general posterior Jesús del Olmo. Fernando Jáuregui (Bardavío "et al”, 2000: 382) ha descrito los habituales encuentros del director Javier Calderón con numerosos periodistas y también fueron frecuentes los encuentros de Jorge Dezcallar y Alberto Saiz con directores de medios. Jáuregui y Cernuda llegaron a publicar sobre Javier Calderón que "su talante abierto y dialogante, su inequívoco sello de demócrata, le granjearon grandes simpatías entre los profesionales de la información".

Todos estos casos divulgados de contactos de periodistas con la dirección del servicio secreto, y otros habituales y frecuentes con otros altos cargos, demuestran que los mandos del CESID antes y el CNI ahora tienen establecida una ancha línea de comunicación directa con la prensa. Mientras públicamente defienden la necesidad de que no se hable de sus actividades, cada vez que lo consideran oportuno se convierten en fuente de información. Bien para contar algunos de sus éxitos, bien para actuar como agentes de influencia.

Ese mismo objetivo, al que podríamos sumar la consideración de venganza o defensa propia -según la óptica desde la que se mire- fue la que llevó a la dirección del servicio secreto a filtrar a los medios de comunicación una información que perjudicaba a uno de sus agentes. Agustín Cassinello fue cesado como Director Técnico de Inteligencia el 12 de noviembre de 2009 por el director del CNI, Alberto Saiz. Nombrado hacía solo cuatro meses, el cese se debió en realidad al enfrentamiento de Cassinello con el Director Técnico de Operaciones, Francisco Montes, por un tema de competencias. Sainz le solicitó que presentara su dimisión y al negarse lo cesó. Tres días después, el 15 de noviembre de 2008, el diario $A B C$ publicó una noticia ofreciendo otra versión: Cassinello había sido destituido por presuntos fallos de información en el ataque de un suicida talibán llevado a cabo el 9 de noviembre en Afganistán, que había costado la vida al brigada Juan Andrés Suárez y al cabo Rubén Alonso. Según el diario El Mundo, que relató pormenorizadamente la historia, "fuentes conocedoras del incidente apuntan a la dirección del CNI como responsable de la filtración al rotativo" (García Abadillo, 2009b: web).

Otro elemento trascendental a tener en cuenta y que demuestra la presencia intencionada de la dirección del servicio secreto en los medios de comunicación es la existencia de campañas de imagen. El 15 de abril de 1997, el director del entonces CESID, Javier Calderón, anunciaba en una reunión con periodistas en el palacio de la Moncloa un plan para cambiar la imagen del Centro (Rueda, 1999: 159). Constaba de tres fases: una entrevista en prensa, un libro y una intervención en televisión. En los meses siguientes, concedió una entrevista al semanario Tiempo, la periodista Pilar Urbano publicó el libro "Yo entré en el CESID" y finalmente hizo unas declaraciones en el programa de televisión "Caiga quien caiga". Paralelamente, llevaron a cabo una política de "puertas abiertas", invitando a visitar su sede a políticos, jueces y periodistas. La idea era demostrar que no tenían nada que ocultar. 


\subsection{Colaboradores e informantes}

Otras fuentes relacionadas directamente con los servicios de inteligencia, aunque con un estatus un tanto peculiar, son los colaboradores e informantes. Personas que ayudan puntualmente con la cesión de una casa o la contratación de una empresa para trabajos especiales, o gente que colabora facilitando información que el servicio necesita para cumplir determinadas misiones. Carecen de la obligación legal de secreto, aunque el servicio de inteligencia confíe en su discreción.

Son informantes que puntualmente, en temas concretos, ofrecen a los periodistas una información de máxima calidad. Dos casos prácticos explican perfectamente la actitud de este tipo de fuentes. En abril de 1988, el ciudadano marroquí Tarik Ouazzani estaba convencido de que no tardaría mucho tiempo en conseguir la nacionalidad española (Rueda y Pradas, 1997: 169). En su escrito de solicitud había explicado que su madre había nacido en Melilla, por lo que interpretaba que reunía la condición establecida por la ley española. Ante el retraso en la concesión, fue a visitar a Juan José Pretel, Subdirector de Nacionalidad en el Ministerio de Asuntos Exteriores, que le aseguró que había algunos problemas que seguro que se solucionarían. Ouazzani no entendió a qué se refería hasta que un agente del entonces CESID se presentó en su casa y le dijo que si quería ser español tenía que hacerles previamente algunos favores. En los meses siguientes tuvo que ayudar en la captación por parte del servicio de inteligencia de una secretaria de la embajada de Marruecos en Madrid y escribirles informes detallados sobre personas que había conocido cuando vivía en Marruecos. Más tarde, le pidieron que intentara facilitarles contactos en el círculo íntimo del rey Hassan, ante lo que se negó en rotundo. Le dio mil vueltas a su complicada situación y solo encontró un camino para conseguir la nacionalidad: recurrió a la prensa y contó cómo el CESID le había chantajeado. Tarik consiguió la nacionalidad española.

Un segundo caso es el protagonizado por María Isabel del Barrio, funcionaria de la Seguridad Social (El Mundo, 2007: web). En 1985 fue contactada por un agente del CESID para que le sacara los datos de determinadas personas en las que el servicio secreto tenía depositado su interés para algunas investigaciones -una acción ilegal-. La mujer accedió y comenzó a recibir unos emolumentos mensuales por su trabajo. Años después, pasó a sacar información -igualmente ilegal- para detectives privados también a cambio de dinero. En 2001, cuando llevaba 16 años colaborando con el servicio de inteligencia, la policía la detuvo al descubrir que vendía información a detectives. María Isabel pidió ayuda a su contacto en el servicio secreto y al no recibirla, decidió destapar el trabajo que había estado haciendo para ellos. Habló con la prensa y describió el trabajo sucio por el que la habían estado pagando, facilitando nombres de empresarios y actrices a los que había investigado su vida laboral por requerimiento del CESID.

\subsection{Fuentes aledañas}

Para llevar a buen puerto las investigaciones de los periodistas en materia de espionaje hay un último tipo de fuentes humanas que hay que cultivar y que son tremendamente productivas. Los temas que tratan los servicios de inteligencia tienen campos en común con otras instituciones. Aunque la lista podría ser muy amplia, convendría 
destacar a los Cuerpos y Fuerzas de Seguridad del Estado, los cuerpos policiales de varias autonomías y los funcionarios del Ministerio de Asuntos Exteriores. Con frecuencia se convierten en fuentes motivados por sentir una invasión de sus competencias por parte del CNI o porque consideran que han jugado sucio con ellos.

Los casos con policías y guardias civiles se dan continuamente, pero fueron especialmente numerosos $-\mathrm{y}$ han sido documentados- durante el enfrentamiento abierto que mantuvieron esas instituciones con el CESID en la década de los 80 por un conflicto de competencias. En esos años aparece publicado en prensa (Aroca, 1984: 38) en distintos momentos el espionaje al que fueron sometidos, entre otros, Jerónimo Arozamena, vicepresidente del Tribunal Constitucional; Francisco Pera Verdaguer, presidente de la Sala Tercera del Supremo; José Luis Corcuera, ministro del Interior; Gregorio Peces Barba, presidente del Congreso de los Diputados; y Alberto Oliart, ministro de Defensa. En casi todos los casos, las historias son similares: policías de escolta han descubierto los seguimientos de agentes del CESID. Las informaciones que se filtran a los medios van contra el servicio de inteligencia y sólo pueden proceder de funcionarios del Ministerio del Interior, molestos con la actuación de sus colegas del espionaje.

Los diplomáticos nunca han tenido ese enfrentamiento abierto, aunque tampoco han tenido reparos en denunciar a espías que no actuaban según las reglas éticas que ellos consideraban imprescindibles. Es el caso del embajador en El Salvador a mediados de los años 80, Fernando Álvarez de Miranda (Rueda, 1997: 73). Un periodista de investigación se puso en contacto con él en el año 1997 para confirmar y ampliar datos sobre una historia en la que estaba trabajando. Un agente del CESID, Vicente López Pascual, había estado en El Salvador durante los años 80 apoyando, nada más y nada menos, que a los escuadrones de la muerte que tanto daño habían llevado al país. El embajador accedió a contar todo lo que sabía y además especificó que él personalmente había telefoneado al director del servicio de inteligencia para quejarse del comportamiento del agente.

\subsection{Fuentes materiales}

Junto a todas estas fuentes personales, cobran una relevancia especial las materiales. Los documentos del CNI tienen por su objetividad y credibilidad un valor destacable para cualquier informador. El inconveniente es que la mayor parte son considerados secretos, aunque por su contenido existen algunos de dudoso encaje en ese concepto. Por ejemplo, el "Manual de inteligencia" que reprodujo el semanario Tiempo (Rueda, 1995), trata de enseñar a los agentes los conceptos teóricos del trabajo del espía, pero no parece contener nada de información susceptible de dañar la seguridad nacional.

Hay documentos que teniendo la consideración de secretos son publicados por los informadores porque consideran que aportan credibilidad a la historia, no ponen en riesgo ni a personas ni a instituciones, y desvelan una información que la opinión pública tiene derecho a conocer.

Este fue el caso descubierto en 1996. Documentos del CESID, acompañados de una cinta, apoyaron una historia sorprendente (Rubio y Cerdán, 1995: 1): en 1978, cuando Adolfo Suárez era presidente del gobierno, visitó la sede del servicio secreto y se reu- 
nió con sus más importante mandos. Allí hablaron sobre todos los temas conflictivos, entre los que estaba la lucha contra ETA, en la que Suárez se mostró claramente partidario de no utilizar la guerra sucia, aunque sus palabras en algún momento pudieron parecer ambiguas. Años después, se dijo que alguien en el gobierno socialista de ese momento pensaba utilizarlas para probar que la UCD apoyó la guerra sucia. La información publicada aportaba la prueba material de la existencia del documento y el contenido de la conversación grabada por mandos del servicio secreto sin el conocimiento del presidente. De ese modo, la manipulación quedó finiquitada.

Otro caso fue el del documento del CESID llamado "Somosierra-Cilindro-Perellón", que recogía la investigación que habían llevado a cabo durante varios años sobre la vida privada y pública de Juan Alberto Perote, ex jefe de los Grupos de Apoyo Operativo (Belloch, 1997: 16). En el informe se aportaban datos obtenidos gracias a registros de su oficina de trabajo, intervenciones telefónicas y seguimientos. El objetivo de su filtración, realizada aparentemente por el propio servicio secreto, pretendía hacer llegar a la opinión pública el mal comportamiento de un antiguo agente que había estado pasando información secreta sobre irregularidades del servicio.

Las otras fuentes materiales habituales en el periodismo de investigación son los archivos públicos, de uso muy interesante y más habitual en esta especialidad de lo que podría pensarse. Son fuentes abiertas y en ellas se puede encontrar, entre otros datos, empresas y pisos que son utilizadas en el CNI para sus actividades clandestinas. Disponiendo previamente de una pista, es fácil obtener los datos registrales y el nombre de los accionistas o propietarios.

Uno de los casos más claros es el que ocurrió en 1983 con el alquiler del piso en el que iba a vivir el ministro de Defensa, Narcís Serra (Rueda, 1991: 18). Preocupados por su seguridad, el director del CESID, Emilio Alonso Manglano, se ofreció a ayudar a su mujer a buscar uno y cuando lo encontraron decidieron que lo alquilara una de sus empresas tapadera, Uzcalar. Defendieron que fue por motivos de seguridad, aunque el hecho de que nunca más en la historia de España un servicio secreto alquilara el piso de un ministro da pábulo a otra teoría: lo utilizaban también para asuntos oscuros, como espiar a algunas de las embajadas cercanas. El hecho es que el periodista recibió la pista sobre la extraña situación y buscó en el registro mercantil los datos de la citada empresa. Descubrió que todos sus cargos directivos eran agentes del servicio, lo que le permitió escribir la historia de denuncia.

\section{Conclusiones}

Las limitaciones impuestas por la Ley de Secretos Oficiales y por la Ley Reguladora del CNI no han impedido el control por parte de los periodistas de las actividades del servicio de inteligencia. $\mathrm{Y}$ ha sido gracias a las numerosas fuentes con que han contado los profesionales, mucho más numerosas de las que podrían pensarse en un primer acercamiento al tema.

Se observa del análisis una tipología de fuentes reseñadas que se concretan en las siguientes: los agentes y ex agentes; los miembros del Gobierno; los parlamentarios; el gabinete de prensa del CNI; la cúpula del CNI; colaboradores e informantes del servicio de inteligencia; las fuentes aledañas; $y$ las fuentes materiales. 
Todas las personas vinculadas de una u otra manera con los asuntos que tratan los servicios de inteligencia pueden verse impelidas a filtrar a la prensa informaciones que conocen o colaborar facilitando datos. Como ha quedado referenciado, las fuentes estudiadas han colaborado en algún momento con los profesionales de los medios de comunicación cuando sus intereses particulares o políticos así se lo recomendaba. Obviando los límites impuestos por las leyes citadas.

La actividad de las fuentes no oficiales despierta malestar en el CNI y el gobierno. Que cualquier persona conocedora de estos asuntos hable con la prensa sin permiso es uno de los mayores pecados que puede cometer. Para ellos son traidores que tratan de dañar al servicio y a los intereses del país. Sin embargo, miembros de los distintos gobiernos y de las cúpulas directivas del servicio secreto se saltan las normas restrictivas cuando lo aconsejan sus propios intereses.

\section{Referencias bibliográficas}

ANASAGASTI, Iñaki (2004): Agur Aznar, memorias de un vasco en Madrid. Madrid, Temas de Hoy.

AROCA, Santiago (1984): "Ministros y periodistas han sido espiados por el CESID", en Tiempo, 19 de noviembre, pp. 38-43.

BARDAVÍO, Joaquín; CERNUDA, Pilar; y JAUREGUI, Fernando (2000): Servicios secretos. Barcelona, Plaza y Janés.

BELLOCH, Santiago (1997): "La verdadera vida del agente Perote", en Tiempo, 7 de julio, pp. 16-22.

CAMINOS MARCET, José María (1997): Periodismo de investigación: teoría y práctica. Madrid, Síntesis.

CERDÁN, Manuel; RUBIO, Antonio (1995): "El CESID grabó con micrófonos ocultos a Suárez mientras hablaba sobre ETA", en El Mundo, 27 de febrero, pp. 1, 6 y 7.

CERDÁN, Manuel; RUBIO, Antonio (1996): "El Gobierno en funciones niega al juez un documento sobre la guerra sucia", en El Mundo, 3 de abril, pp. 1 y 7.

COUSIDO, María del Pilar (1994): "Libertad de información y seguridad nacional". Revista Española de Defensa, junio de 1994, p. 42.

DADER, José Luis (2002): Periodismo de precisión, vía socioinformática de descubrir noticias. Madrid, Síntesis.

DÍEZ, Anabel (2004): "Zapatero acusa al Gobierno de utilizar los servicios secretos para atacar al PSOE", en El pais: http://elpais.com/diario/2004/01/30/espana/1075417203_850215.html [Consulta: 12-11-2012]

DEL PINO, Javier (2005): “Garganta profunda, ¿deber o venganza?”, en El País, 2 de junio, p. 8.

D.M. y J.P. (2004): "El Gobierno desclasifica papeles del 11-M para intentar demostrar que no mintió en la crisis", en $A B C, 19$ de marzo, pp. 12-14. 
"EL CHIVATO" (2010): "La medalla a "El Lobo", enemigo público número uno de ETA, y los silencios del CNI", en El Confidencial Digital: http://elconfidencialdigital.com/seguridad/049941/la-medalla-a-el-lobo-enemigo-publico-numero-unode-eta-y-los-silencios-del-cni?IdObjeto=24791 [Consulta: 18-04-2013]

EL MUNDO (2007): "Voy a ir a la cárcel por trabajar para el Estado", en El mundo: http://www.elmundo.es/elmundo/2007/08/12/espana/1186890142.html [Consulta: 3-01-2013]

FERNÁNDEZ, J.M. (1992): "Garganta profunda, el misterio", en El Mundo, 12 de junio, Suplemento de Comunicación, p.3.

FUMERO, Javier (2013): "Espionaje español a puerta cerrada", en El Confidencial Digital: http://blogs.elconfidencialdigital.com/javierfumero/2013/03/20/espionajeespanol-a-puerta-cerrada/ [Consulta: 20-03-2013]

GARCÍA ABADILLO, Casimiro (2009a): "Saiz somete a 10 agentes del CNI a la máquina de la verdad", en El Mundo, 22 de junio, pp. 1, 3, 4 y 5.

GARCÍA ABADILLO, Casimiro (2009b): "El cuestionado jefe de los espías", en $E l$ Mundo: $\quad$ http://www.elmundo.es/opinion/columnas/casimiro-garcia-abadi1lo/2009/01/2580345.html [Consulta: 18-12-2012]

GÓMEZ MENDOZA, Miguel Ángel (2000): “Análisis de contenido cualitativo y cuantitativo: Definición, clasificación y metodología". Revista de Ciencias $\mathrm{Hu}$ manas, mayo de 2000, número 20: http://www.utp.edu.co/ chumanas/revistas/revistas/rev20/gomez.htm [Consulta: 12-03-2013]

GONZÁLEZ, Miguel (2013): "El jefe del CNI acusa a la Generalitat de identificar a sus agentes en el extranjero", en El Pais, 20 de marzo, p. 17.

MOLINA, Jesús (2004): "Carod-Rovira ultima un pacto con ETA para que no perpetre atentados en Cataluña", en $A B C, 26$ de enero, PP. 1, 10 y 11.

MUNIZAGA, Giselle (2008): "Uso frecuente de fuentes no identificadas: la permanencia del fenómeno", en Observatorio Fucatel: http:/www.observatoriofucatel.cl/index.php? $\mathrm{s}=$ estudio + sobre + uso + de + fuentes + no + identificadas + en + la + prens a+chilena. [Consulta: 12-11-2012]

PÉREZ OLIVA, Milagros (2010): “Demasiadas fuentes anónimas”, en El País: http://elpais.com/diario/2010/09/26/opinion/1285452005_850215.html [6-10-2012]

REIG, Ramón (2000): Periodismo de investigación y pseudoperiodismo. Madrid, Ediciones Libertarias.

RODRÍGUEZ, Pepe (1994): Periodismo de investigación: técnicas y estrategias. Barcelona, Paidós.

RUBIO, Antonio (2009a): “Agentes del CNI dicen que el director utiliza el centro en su propio beneficio", en El Mundo, 14 de abril, p. 6.

RUBIO, Antonio (2009b): "Acusan al director del CNI de pescar en Senegal con dinero público", en El Mundo, 16 de junio, pp. 4 y 5. 
RUEDA, Fernando (1991): "Serra vive en un piso del servicio secreto", en Tiempo, 2 de diciembre, pp. 18-20.

RUEDA, Fernando (1995): "Manual de inteligencia del CESID", en Tiempo, 3 de julio, libro entregado conjunta e inseparablemente con el número del semanario.

RUEDA, Fernando (1995): "Un asesino a sueldo, listo para matar a Perote", en Tiempo, 16 de octubre, pp. 16- 22.

RUEDA, Fernando y PRADAS, Elena (1997): KA: licencia para matar. Qué hacen y cómo son los espías más peligrosos del CESID. Madrid, Temas de Hoy.

RUEDA, Fernando (1999): Por qué nos da miedo el CESID. Madrid, Foca.

RUIZ OLABUÉNAGA, José Ignacio (2012): Metodología de la investigación cualitativa. Pamplona, Universidad de Deusto.

SAINZ DE LA PEÑA, José Antonio (2001): "Los servicios de inteligencia del Magreb", en MACIÁ, Juan: Periodismo y servicios secretos. Madrid, Universitas.

SÁNCHEZ, Manuel (2009): "Saiz denuncia una "venganza" de agentes del antiguo CESID", en El Mundo, 24 de junio, pp. 1, 3, 4 y 5.

SECANELLA, Petra (1986): Periodismo de investigación. Madrid, Tecnos.

SEIJAS, Leopoldo (2001): “La CIA y los medios de comunicación”, en MACIÁ, Juan: Periodismo y servicios secretos. Madrid, Universitas.

SORIA, Carlos (1997): El laberinto informativo: una salida ética. Pamplona, EUNSA.

WAISBORD, S. (2001): "Por qué la democracia necesita del periodismo de investigación", en Sala de Prensa: www.saladeprensa.org/art231.htm. [Consulta: 16-062005] 